Mots. Les langages du politique

Le rapport, entre description et recommandation

\title{
Les rapports d'initiative au Parlement européen ou comment la traduction influe sur les aspects performatifs d'un genre discursif
}

Own-initiative reports to the European Parliament or how translation affects the performative aspects of a discursive genre

Los informes de iniciativa en el Parlemento europeo o la influencia de la traducción sobre los aspectos performartivos de un género discusivo

\section{Rachele Raus}

\section{OpenEdition}

Journals

Édition électronique

URL : https://journals.openedition.org/mots/22810

DOI : $10.4000 /$ mots. 22810

ISSN : 1960-6001

Éditeur

ENS Éditions

Édition imprimée

Date de publication : 10 juillet 2017

Pagination : 95-115

ISSN : 0243-6450

Référence électronique

Rachele Raus, « Les rapports d'initiative au Parlement européen ou comment la traduction influe sur les aspects performatifs d'un genre discursif », Mots. Les langages du politique [En ligne], 114 | 2017, mis en ligne le 10 juillet 2019, consulté le 23 avril 2022. URL : http://journals.openedition.org/mots/ 22810 ; DOI : https://doi.org/10.4000/mots.22810

\section{(C) ENS Éditions}




\section{Les rapports d'initiative au Parlement européen ou comment la traduction influe sur les aspects performatifs d'un genre discursif}

En tant que discours produit par l'«institution» (Oger et Ollivier-Yaniv, 2003a, p.127), le rapport d'initiative du Parlement européen permet de questionner, entre autres, l'autorité discursive (Monte et Oger, 2015), la ritualisation de l'énonciation (Krieg-Planque et Oger, 2010, p. 92-93), la présence d'un «ton » spécifique (Maingueneau, 2002, p.127), la relation à l'éthique (Koren, 2008)... Parmi les dimensions contribuant à délimiter les contours d'un genre discursif particulier, l'« efficacité de la parole légitime» (Krieg-Planque, 2012, p.48), et par là la visée prescriptive et/ou incitative du discours, s'accompagne d'un processus de « lissage énonciatif» (Oger et Ollivier-Yaniv, 2006, p. 64). Celui-ci consiste en un aplatissement de la dimension argumentative contribuant à la neutralisation d'un discours dépourvu de toute dimension conflictuelle (Cussò et Gobin, 2008, p. 8).

L'étude des rapports portant sur un sujet conflictuel comme la prostitution, qui ont été rédigés en français et en italien entre 2004 et 2014, permet ici de prolonger l'analyse de cette dynamique discursive. Constitutivement «multilingues ", les rapports d'initiative donnent l'occasion d'aborder, par la comparaison des différentes versions linguistiques, le rôle de la traduction dans la dynamique prescription/incitation-lissage réalisée différemment ou non dans ces deux langues. Nous pourrons ainsi analyser si et comment les traducteurs doublent l'instance énonciative première et peuvent jouer un rôle déterminant ou non à ce propos. Nous mettrons plus particulièrement en lumière leur contribution à la construction différente des effets pragmatiques dans les discours français et italien.

Partant du constat que tout genre de discours est un « dispositif de communication à la fois social et verbal, historiquement défini » (Maingueneau, 2002, p.119), nous avons analysé d'abord les conditions de production spécifiques des rapports d'initiative parlementaire. Ensuite, après avoir présenté notre corpus et le contexte politique concernant le débat parlementaire sur la prostitution, nous avons mené, sur les versions françaises et italiennes des 
rapports, une analyse contrastive des verbes à visée prescriptive et incitative et des modalités d'ancrage énonciatif. La perspective contrastive adoptée a permis de tirer des conclusions à l'égard des effets performatifs de ce genre discursif en relation avec la traduction et avec la manière dont celle-ci influe sur la dynamique prescription/incitation-lissage.

\section{Conditions de production des rapports parlementaires}

Genre «routinier» (Maingueneau, 2007, p. 30), le rapport d'initiative, qui permet au Parlement européen de prendre l'initiative politique concernant tel ou tel sujet, se rapproche des actes juridiques en ce qu'il est fortement « ritualisé » (Maingueneau, [2015], p. 2) et laisse donc des marges de variation limitées lors de sa rédaction et de sa traduction.

Le Règlement interne du Parlement européen (Europe, 2004 et 2009) précise la structure des rapports de l'institution et les procédures de votation et d'approbation : à l'égard du rapport d'initiative, il prévoit une proposition de résolution sur un sujet de compétence des commissions parlementaires, qui sera accompagnée d'un exposé des motifs. La proposition de résolution se compose normalement de trois parties : les visas, les considérants et les dispositions, ces dernières représentant la partie performative du texte par excellence (voir fig. 1) où le Parlement européen affirme ce qu'il pense du sujet en question et s'engage ou demande l'engagement des autres acteurs concernés.

Avant d'aboutir au texte final, le rapport est présenté sous la forme d'un projet rédigé dans l'une des 24 langues officielles choisie par le rapporteur, qui le plus souvent préfère en fait s'exprimer en anglais véhiculaire - plus rarement, en français ou en allemand. Ensuite, les parlementaires soumettent leurs amendements dans l'une des langues officielles ${ }^{1}$, qui est normalement mais pas exclusivement leur langue maternelle. Le processus de rédaction de ces textes les rend donc constitutivement multilingues.

Une fois les amendements acceptés ou refusés, le texte final est voté et approuvé ou, plus rarement, rejeté par la commission. S’il est approuvé, il est ensuite déposé et soumis à la discussion et à l'approbation du Parlement européen. La procédure allant de la prise en examen du rapport jusqu'à son adoption finale prend normalement de deux à six mois, à quelques exceptions près. La durée de la procédure varie en raison de plusieurs paramètres : entre autres, la demande d'avis éventuelle présentée à d'autres commissions

1. Le Parlement européen compte 24 langues officielles (voir Europe, 2004, art. 138 et 2009, art. 146). À la différence des projets de rapport qui le plus souvent sont rédigés dans l'une des trois langues de travail le plus utilisées - français (FR), anglais (EN), allemand (DE), les amendements sont souvent proposés dans la langue maternelle du député européen. Les amendements sont votés par les membres de la commission parlementaire concernée. 


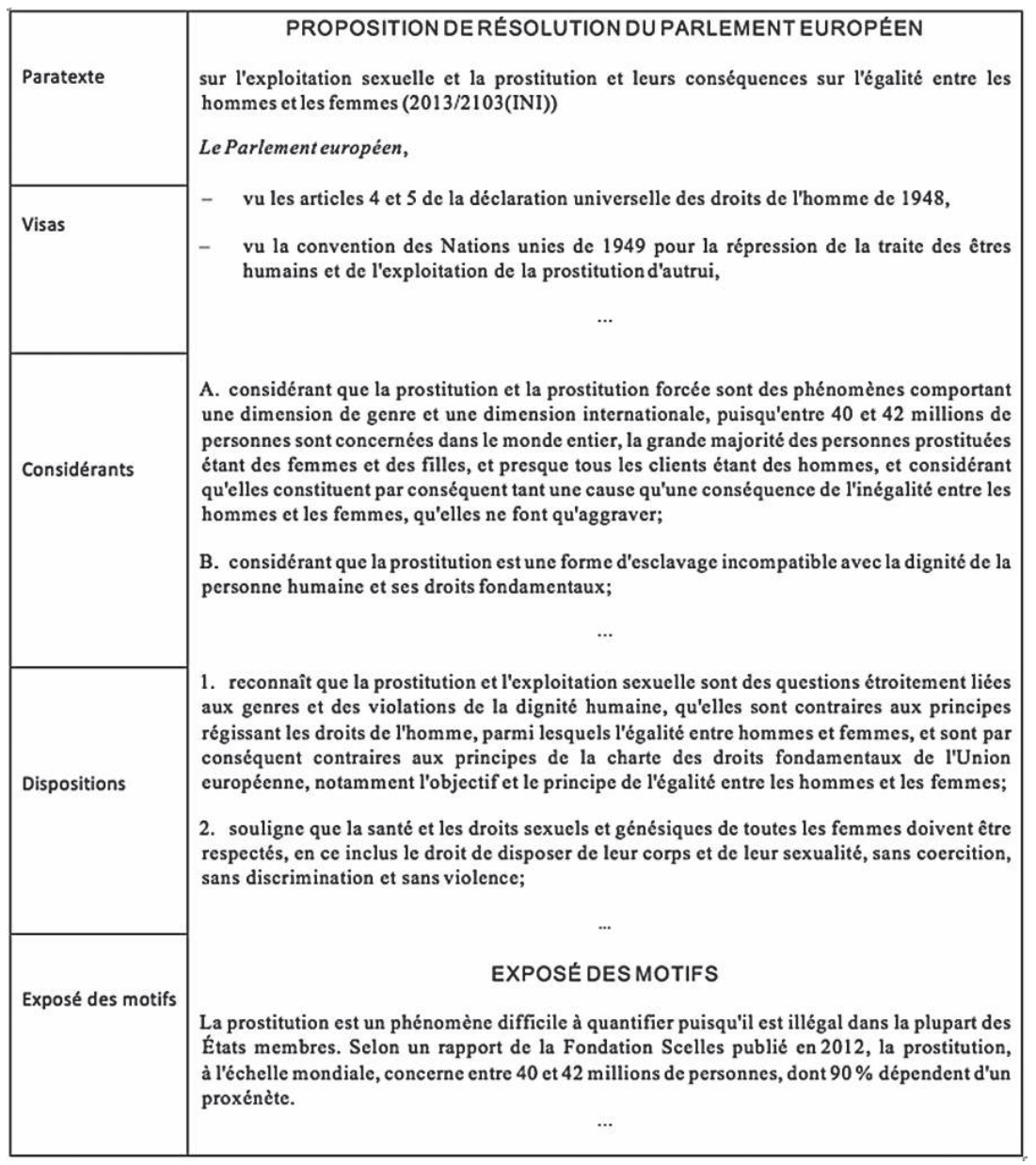

Figure 1 : Structure du rapport d'initiative

parlementaires et surtout le nombre d'amendements présentés. Dans notre corpus, le rapport de l'Autrichienne Christa Prets (2005) est approuvé dans le délai le plus court. C'est aussi celui qui a obtenu le plus de votes pour (622) et le moins de votes contre lors de la votation finale en assemblée. À l'inverse, le rapport de l'Anglaise Mary Honeyball (2014) a attendu environ huit mois avant d'être voté et approuvé par le Parlement européen et a subi plusieurs amendements en commission, et n'a finalement pas été voté à l'unanimité, comme le montre la présence d’opinions minoritaires à la fin du document ${ }^{2}$. La traduction

2. La présence des avis minoritaires est d'ailleurs prévue par le Règlement du Parlement européen lorsque l'avis de la commission concernée n'est pas unanime (voir Europe, 2004, art. 46 et 2009, art. 52). 
des rapports d'initiative, qui dans leur version finale sont rendus disponibles dans la plupart des langues officielles, est normalement faite par le traducteur de l'institution. Le recours aux traducteurs externes peut concerner notamment la partie du texte qui reste la plus libre à traduire, à savoir l'exposé des motifs3. Cette partie n'est pas soumise à votation et se caractérise par :

la tension constitutive entre des contraintes rédactionnelles et intertextuelles fortes, d'une part, et, de l'autre, la présence du travail multilingue qui décentre constamment l'effort centripète à l'harmonisation par la tentative d'adapter le texte à la langue d'arrivée. (Raus, 2013, p. 62)

Traducteurs internes ou externes à l'institution partagent un certain nombre de caractéristiques communes comme le fait d'utiliser des logiciels d'aides à la traduction (notamment le logiciel SDL Trados), de disposer de mémoires de traduction 4 et d'outils partagés (guides rédactionnels, entre autres). L'utilisation croissante des mémoires de traduction et la reprise intertextuelle caractérisant ces documents, notamment la partie initiale des visas, ainsi que la tendance des rapporteurs à s'appuyer souvent sur des argumentaires communs, entraînent une automatisation de la traduction de plus en plus évidente.

Malgré la présence d'un processus marqué par la standardisation, l'automatisation et la bureaucratisation, les documents finaux demeurent perméables à des remaniements multilingues. Ainsi, il arrive parfois que la traduction prime sur l'original (Leoncini Bartoli, 2011, p. 231), ce qui porte à parler de la présence d'une instance énonciative «double» (énonciateurs et/ou traducteurs). Ce constat permet d’insister sur la présence d'énonciateurs multiples et, par conséquent, de locuteurs différents qui contribuent à la construction de la visée pragmatique du texte.

\section{Le corpus et la méthode d’analyse}

Notre corpus d'analyse se compose des versions françaises et italiennes de onze rapports d'initiative concernant la prostitution, rédigés entre 2004 et 2014 par la Commission des droits de la femme et de l'égalité des chances. Cette commission devient, à partir de la 6e législature de 2004, la Commission de la femme et de l'égalité des genres5. Nous avons retenu non seulement les docu-

3. Raus, 2010, p.117-118. Dans notre corpus, les parties traduites par des traducteurs externes sont les exposés des motifs des rapports italiens d'Anne Karamanou (2004), de Christa Prets (2005), de Véronique De Keyser (2006) et l'ensemble du projet de rapport de Maria Carlshmare de 2008.

4. Pour un recensement exhaustif des ressources numériques multilingues du Parlement européen et de l'Union, entre autres des mémoires de traduction rendues disponibles via le Joint Research Centre, voir Ralf Steinberger et alii (2014).

5. Les documents de notre corpus sont disponibles sur le site <http://www.europarl.europa.eu/ portal/fr> (consulté le 24 mars 2017). 
ments portant sur la prostitution de manière explicite mais aussi les rapports où le terme prostitution est présent de manière significative. Nous avons donc écarté les rapports où le terme reste un hapax ou est très peu fréquent (deux, trois occurrences). Le corpus rassemble les rapports finaux approuvés par la commission, à l'exception du rapport présenté par la Suédoise Marie Carlshamre, qui a été rejeté en novembre 2008; nous n'avons alors pu prendre en compte que le projet de la députée.

Nous avons analysé de manière contrastive les versions françaises (81 296 mots) et italiennes (74196 mots) des rapports. On l'a vu, ce type de document est constitutivement multilingue, la plupart des projets des rapports sont produits en anglais langue maternelle ou véhiculaire, et plus rarement en français ou en allemand, avant de subir des amendements multilingues ${ }^{6}$. Par conséquent, les versions françaises et italiennes sont très souvent des traductions à partir de l'anglais, d'où l'importance de faire un détour par les versions anglaises (EN) des documents pour comprendre les raisons de certaines traductions.

À la différence de la plupart des recherches existantes sur des corpus multilingues, qui prennent en considération des corpus comparables exploités par des outils spécifiques (voir, entre autres, Ho-Dinh, Valette, 2014), nous voulions analyser un corpus de textes alignés (FR/IT) de manière contrastive. Un travail de ce type implique non seulement le recours à des concordanciers ou, dans une approche textuelle, à des outils de textométrie, mais, là où c'est possible, le développement de supports informatiques sur mesure (voir Hermand, 2014).

Pour le dépouillement du corpus, nous avons donc utilisé le logiciel AntPConc (Anthony, 2014) qui non seulement indique les fréquences et les cooccurrences mais permet également d'afficher les corpus parallèles de différentes langues. Ce logiciel n'alignant pas les textes, nous avons également utilisé le logiciel de traduction SDL-Trados Studio 2015 afin d'aligner correctement les documents de manière à créer une mémoire de traduction. Une comparaison ponctuelle des segments textuels 7 ainsi que des reprises intertextuelles 8 a été réalisée. En général, l'approche quantitative est allée de pair avec une approche qualitative attentive aux utilisations discursives mais aussi et surtout aux réalisations discursives de la traduction.

6. À la différence des projets et des rapports finaux, les amendements indiquent la langue originale des modifications demandées.

7. Par «segment textuel » nous entendons les paragraphes du texte tels qu'ils sont segmentés dans Trados lors de l'alignement des rapports.

8. Nous utilisons volontairement la notion d'intertexte (voir Paveau, 2010) parce qu'elle est constitutive de l'utilisation des mémoires de traduction. Cette pratique est fondée sur la reprise et la recontextualisation dans d'autres rapports de segments textuels spécifiques préexistants répertoriés. Elle caractérise non seulement la traduction mais aussi la rédaction des rapports qui reprennent en partie des segments textuels, plus particulièrement dans la partie des visas. 


\section{Le débat sur la prostitution au Parlement européen et le contexte politique}

Dans notre corpus, la prostitution est mise normalement en relation avec d'autres phénomènes négatifs comme la guerre, la traite, la pauvreté, les inégalités, la violence ou encore la criminalité. Elle évoque notamment des univers sémanticosymboliques (Cabasino, 2001, p.104) qui vont être des lieux de conflits entre les abolitionnistes (notamment Espagne et Suède) et les réglementaristes (surtout Pays-Bas, Allemagne et Autriche) dès $2004^{9}$ : dans le rapport de la Suédoise Marianne Eriksson (2004), le discours des réglementaristes conteste l'amalgame de la prostitution avec la traite, amalgame qui est par contre soutenu par les abolitionnistes dans le rapport Honeyball (2014, p.16). Ou encore, la dissociation qui s'opère entre «prostitution forcée» et "prostitution légale» caractérise le discours des réglementaristes dès 2004 et s'oppose à la négation de la professionnalisation de la prostitution dans le discours des abolitionnistes.

Dans un témoignage à propos de l'action de la Commission des droits de la femme et de l'égalité de genre entre 2009 et 2014, la députée européenne Sylvie Guillaume, membre de cette commission, a justement souligné la présence de points de vue qui ne sont pas nivelés par l'accord général sur la nécessité de promouvoir l'égalité, puisque «chacun a une couleur politique et défend des principes et des priorités» (Toutain, 2013, p. 36). Un document sur la prostitution rédigé pour la Direction générale des politiques internes du Parlement (Schulze et alii, 2014) parle de tensions constitutives sur ce sujet dès les années 1990, non seulement entre les États membres, qui ont des politiques différentes, mais aussi à l'égard des groupes d'intérêts et des associations qui se sont organisés à l'international et qui ont une influence grandissante sur l'agenda politique des institutions européennes (voir aussi Saurugger, 2003). Les groupes de pression dans ce domaine sont notamment le Lobby européen des femmes (LEF), qui plaide pour une approche abolitionniste, et les syndicats des travailleuses du sexe organisées dans le Syndicat international des travailleurs du sexe (IUSW), qui revendique l'approche règlementariste et compte sur l'appui de féministes défendant l'autodétermination des femmes. La campagne entamée par le LEF depuis 2011 contre la prostitution, qui débouchera sur l'appel de Bruxelles de 2012 porté par 200 ONG, est fondamentale en ce sens : le 2 octobre 2013, les députés européens signent à leur tour cet appel.

Les tensions entre règlementaristes et abolitionnistes, tout comme l'évolution vers le choix (néo-)abolitionniste final ${ }^{10}$, sont «visibles dans le débat»

9. L'Italie et la France ont tendance à soutenir le modèle suédois pendant les travaux parlementaires.

10. Le fait de parler de choix «néo-abolitionniste» découle de la prise en compte du modèle sué- 
parlementaire (Schulze et alii, 2014, p. 29) et laissent des traces dans la surface textuelle des rapports : si le rapport Eriksson fait coexister de manière incohérente les arguments abolitionnistes et réglementaristes (Raus 2015, p. 126) en 2004, dix ans plus tard, lorsque la position abolitionniste l'emportera, les arguments réglementaristes resteront confinés dans les avis minoritaires à la fin du rapport Honeyball (2014). C'est justement parce qu'il marque un tournant événementiel dans l'action politique européenne à l'égard de la prostitution que ce dernier rapport, publié vers la fin de la $7^{\mathrm{e}}$ législature du Parlement européen, nous a permis de fixer un terme à l'analyse.

La présence d'un sujet conflictuel rend encore plus intéressant l'analyse de ces documents. On peut ainsi voir de quelle manière la visée prescriptive/ incitative des rapports est plus ou moins atténuée lors de la traduction et dans quelle mesure peut donc s'opérer un processus de lissage énonciatif. C'est ce que nous allons maintenant analyser de près.

\section{L’analyse des rapports entre visées prescriptive/incitative et lissage}

Afin de retracer la dynamique prescription/incitation - lissage de manière contrastive dans les versions françaises et italiennes des rapports concernés, nous avons estimé utile de regarder de près d'une part les verbes à visée prescriptive et incitative et de l'autre, la façon dont les modalités d'ancrage énonciatif se réalisent dans les deux versions linguistiques des rapports prises en considération.

\section{L'utilisation des verbes et l'engagement des acteurs}

\section{Les verbes modaux à visée prescriptive}

L'analyse a porté tout d'abord sur la manière dont il est possible de remarquer des décalages entre les discours français et italiens par rapport à leur pouvoir d'imposer l'action. À cet égard, nous avons pris en considération l'utilisation du verbe français devoir et de l'équivalent italien dovere ${ }^{11}$. Le fait d'avoir choisi d'analyser ce verbe modal à la place d'autres verbes français à visée prescriptive comme falloir ou de la locution verbale il est nécessaire est dû au fait que :

dois qui incrimine le client et essaie de « récupérer» la personne prostituée. Faisons remarquer que trois des dix rapporteurs de notre corpus sont des députées suédoises.

11. Nous n'avons pas tenu compte des occurrences à visée épistémique ou aléthique (Vetters, 2004), notre but étant justement d'observer les mécanismes d’obligation théorique (morale) et pratique (matérielle). 
par rapport à falloir ${ }^{12}$, devoir est le verbe normalement utilisé pour traduire l'anglais must et should (Tron, 2010, p.18) qui restent les verbes modaux à visée prescriptive qui sont normalement utilisés dans les documents en anglais;

à la différence des locutions il est nécessaire - è necessario, qui sont utilisées au présent de l'indicatif13, devoir - dovere sont utilisés aussi au conditionnel et permettent de mieux retracer les décalages entre le français et l'italien sur le plan de l'atténuation de l'engagement demandé.

L'analyse lexicométrique permet de dresser un premier bilan à l'égard des tendances différentes des versions françaises et italiennes des rapports :

\begin{tabular}{|c|c|c|}
\hline Verbe modal & Fr. (tot. 173 occurrences) & It. (tot. 133 occurrences) \\
\hline Doit - doivent & $49,13 \% a$ & \\
\hline Deve - devono & & $43,6 \%$ \\
\hline Devrait - Devraient & $50,87 \%$ & \\
\hline Dovrebbe - Dovrebbero & & $56,4 \%$ \\
\hline
\end{tabular}

Tableau 1 : Le verbe modal devoir/dovere dans les rapports d'initiative

Le tableau 1 permet de constater que le traducteur italien a tendance à affaiblir la visée prescriptive par l'utilisation du conditionnel. Remarquons également que la version italienne utilise moins le verbe dovere par rapport à l'équivalent français devoir des rapports français (133 occurrences de dovere contre 173 de devoir), et cela pour deux raisons. D'abord, le traducteur italien utilise également des verbes synonymiques (ex. bisognare, consentire, occorrere) qui pourtant pourraient s'avérer plus faibles du point de vue performatif14. Ensuite, le traducteur italien semble privilégier des traductions littérales de l'anglais et cela à la différence du français. C'est le cas de l'énoncé «a country which does not consider murder to be a criminal offence could not become a member» [c'est nous qui soulignons en gras] tiré du rapport Carlshamre (2005) et qui est ainsi traduit dans les deux langues:

(FR) ... un pays qui, par exemple, ne considère pas le meurtre comme un délit ne devrait pas pouvoir devenir membre de l’Union européenne.

12. Il faut/ il faudrait n'apparaissent que six fois au total dans notre corpus.

13. L'expression «X serait nécessaire» est absente du corpus et l'équivalent italien « $X$ sarebbe necessario » n'est présent que deux fois.

14. Précisons que le peu de cas d'utilisation de ces verbes (occorrere onze occurrences; bisognare deux occurrences; essere opportuno huit occurrences; essere necessario sept occurrences) concernent des prescriptions pratiques qui sont normalement rendues en français par le conditionnel de devoir et en anglais par should (be). 
(IT)... un paese per il quale l'omicidio non è da considerarsi un reato penale non potrebbe divenire membro dell'Unione.

Le verbe italien potrebbe est en effet la traduction littérale de l'anglais could, qui par contre devient devrait en français. Remarquons que le traducteur italien choisit de traduire de manière littérale aux dépens de la lisibilité du texte ${ }^{15}$.

La tendance italienne à affaiblir la visée prescriptive et l'engagement des acteurs ressortirait aussi de la présence des acteurs en cooccurrence avec le modal concerné, comme on peut le constater dans le tableau 2 :

\begin{tabular}{ll}
\hline Fr. & It. \\
\hline UE/Union européenne & UE/Unione europea \\
\hline État(s) (membre-s) & Stato/i (membro/i) \\
\hline Membres & Membri \\
\hline Gouvernement & Governo \\
\hline Personnes/Individu & Persone \\
\hline Femmes/elles & Donne \\
\hline Personnes prostituées & Prostitute \\
\hline Victimes & Vittime \\
\hline Criminels/Coupables & Responsabili \\
\hline Parlement & $\varnothing$ \\
\hline Société & $\varnothing$ \\
\hline Tribunaux & $\varnothing$ \\
\hline
\end{tabular}

Tableau 2 : Les acteurs principaux du verbe modal devoir/dovere dans les rapports d'initiative

Remarquons la tendance de l'italien à ne pas engager des acteurs comme le Parlement, la société, les tribunaux... et à utiliser parfois des euphémismes (responsabilivs coupable/criminel, alors que les équivalents parfaits colpevole/criminale existent). Il est vrai que, dans un tel contexte, l'utilisation du terme neutre responsable serait plus approprié en français aussi, puisque les deux autres termes supposent la condamnation d'un tribunal. Dans des cas comme celui-ci, les traducteurs sont autorisés à «neutraliser» les connotations du texte.

Cependant, la tendance du traducteur italien est de moins engager les acteurs concernés en général : bien que l'italien reste une langue réceptive (Caliendo, 2004, p. 9), l'analyse révèle que la version italienne se démarque de celle en anglais, sans doute l'originale, et de celle en français quand il

15. La lisibilité implique que le document traduit non seulement soit facile à lire mais aussi que le lecteur ne le perçoive pas comme le résultat d'une traduction. 
s'agit de se positionner ${ }^{16}$ par rapport aux acteurs et à leur engagement, et tend à privilégier l'abstraction et les acteurs non animés. Par exemple, voici ce qui arrive dans le rapport Eriksson de 2004, dont nous transcrivons ici trois extraits (tableau 3) :

En.

Fr.

It.

This is why many users who C'est ainsi que de nomare interested in paedophilia breuses personnes enter certain chatrooms for s'intéressant à la pédophilie children and young people in se tournent vers certains order to get in touch perso- forums de discussion nally at a subsequent time. destinés aux enfants et This is where 'employment aux jeunes, de manière à agencies' can also be found. pouvoir ensuite établir des contacts personnels. Dans ce contexte aussi existent des «intermédiaires».
Per questo, molte persone con interesse per la pedofilia ricercano determinati spazi di "chat" per bambini e adolescenti, per potersi mettere poi in contatto diretto. In questo modo si trovano anche "intermediazioni di lavoro"
The media's categorisation of the audience primarily as sexual beings has an increasingly significant bearing on the content and its target audience.
Le classement du public par les médias en tant qu'êtres avant tout sexués revêt une importance d'autant plus grande pour le contenu et le public visé.
La categorizzazione del pubblico, compiuta dai mezzi di informazione, indica che gli oggetti sessuali hanno sempre una grande importanza per il loro contenuto e l'orientamento del pubblico
On the Internet images and video clips can be found both free of charge and for payment. The porn companies produce everything
Sur Internet, il est possible In Internet si trovano de trouver des photos et des immagini e videoclip, clips, à la fois gratuitement gratuitamente e a pagaet contre paiement. Les entreprises de pornographie produce tutto produisent de tout

\section{Tableau 3 : L'abstraction des acteurs italiens dans le rapport Eriksson (2004)}

Le tableau montre en effet que le traducteur italien privilégie la nominalisation dépersonnalisée : dans la version italienne, l'intermédiation sur le marché du travail (intermediazione di lavoro) remplace les intermédiaires/les employmentagencies, les objets sexués (oggetti sessuali) substituer aux êtres sexués/le sexual beings et l'industrie du porno (industria del porno) prend la place des entreprises de pornographie/porn companies. Soulignons que la modulation industria del porno pourrait se justifier par l'adaptation linguistique, mais que l'utilisation des oggetti sessuali et des intermediazioni del lavoro relève plutôt d'un choix de traduction. Ajoutons que la base de données terminologiques de l'Union européenne (IATE) n'est pas d'une grande aide pour la traduction de ces termes.

16. Par positionnement, il faut entendre ici la position qu' « occupe un locuteur dans un champ de discussion » (Maingueneau et Charaudeau, 2002, p. 453) ainsi que sa manière de délimiter un lieu de production discursive précis à l'aide de stratégies lexicales et discursives. 


\section{Les verbes dans les dispositions des rapports}

Dans la partie des dispositions du rapport (cf. supra, fig. 1), chaque paragraphe numéroté est introduit par un verbe par lequel le Parlement européen peut soit inciter à l'action les autres acteurs, notamment la Commission, le Conseil et les parties concernées (par exemple demande, recommande, encourage...), soit se positionner face à l'objet du discours et aux acteurs sollicités (par exemple estime, considère, note...). Par conséquent, ces verbes sont particulièrement intéressants pour notre analyse.

Ces verbes peuvent être regroupés plus généralement en fonction de leur visée contextuelle (tableau 4):

\begin{tabular}{lll}
\hline Visée du verbe & \multicolumn{1}{c}{ Fr. } & \multicolumn{1}{c}{ It. } \\
\hline Exposition d'une idée & Constate, observe, reconnaît & Osserva, riconosce, prende atto \\
\hline Défense d'une position & $\begin{array}{l}\text { Considère, estime, note, } \\
\text { rappelle }\end{array}$ & $\begin{array}{l}\text { Considera, nota, rammenta, } \\
\text { ribadisce, richiama, ricorda, } \\
\text { rileva, ritiene, sostiene }\end{array}$ \\
\hline $\begin{array}{lll}\text { Mise en valeur d'une } \\
\text { idée }\end{array}$ & $\begin{array}{l}\text { Appelle, attire l'attention, } \\
\text { insiste, met l'accent, souligne }\end{array}$ & $\begin{array}{l}\text { Evidenzia, insiste, pone } \\
\text { l'accento, sottolinea }\end{array}$ \\
\hline Critique & Condamne, exige, s'inquiète & $\begin{array}{l}\text { Condanna, deplora, esige, si } \\
\text { rammarica }\end{array}$ \\
\hline $\begin{array}{l}\text { Proposition d'une } \\
\text { solution (incitation à } \\
\text { l'action) }\end{array}$ & $\begin{array}{l}\text { Demande, encourage, } \\
\text { exhorte, invite propose, } \\
\text { recommande, suggère }\end{array}$ & $\begin{array}{l}\text { Chiede, esorta, incoraggia, } \\
\text { invita, propone, raccomanda, } \\
\text { suggerisce }\end{array}$ \\
\hline Approbation & Salue, se félicite & Accoglie, plaude, si compiace \\
\hline
\end{tabular}

Tableau 4 : Types de verbes introduisant les paragraphes des dispositions des rapports d'initiative

Voici quelle est leur fréquence d'utilisation dans les rapports en français et en italien (tableau 5) :

\begin{tabular}{lll}
\hline \multicolumn{1}{c}{ Visée du verbe } & \multicolumn{1}{c}{ Fr. (tot. 697 occurrences) } & \multicolumn{1}{c}{ It. (tot. 648 occurrences) } \\
\hline Exposition d'une idée & $34(4,87 \%)$ & $35(5,4 \%)$ \\
\hline Défense d'une position & $77(11,04 \%)$ & $103(15,89 \%)$ \\
\hline Mise en valeur d'une idée & $183(26,25 \%)$ & $121(18,67 \%)$ \\
\hline Critique & $22(3,15 \%)$ & $34(5,24 \%)$ \\
\hline $\begin{array}{l}\text { Proposition d'une solution } \\
\text { (incitation à l'action) }\end{array}$ & $375(53,8 \%)$ & $327(50,46 \%)$ \\
\hline Approbation & $6(0,86 \%)$ & $28(4,32 \%)$ \\
\hline
\end{tabular}

Tableau 5 : Fréquence des verbes introduisant les dispositions dans les rapports d'initiative (en gras les différences majeures) 
Le nombre total des verbes plus faible en italien peut être expliqué. Les traducteurs italiens ont tendance à faire des phrases plus longues, en soudant des segments phrastiques qui restent coupés en français par le point et la virgule et qui demandent par conséquent la répétition du verbe. Par contre, le nombre total de ces verbes en anglais est presque le même par rapport au chiffre français (695 verbes anglais et 697 verbes français au total).

Le tableau 5 permet de constater que la version italienne diffère beaucoup de la version française : l'énonciateur-traducteur italien a tendance à se mettre en relation avec les autres acteurs sollicités, par rapport auxquels il défend sa propre position, critique un peu plus les autres positionnements ou les approuve. Le dit reste polémique, ouvert aux autres en raison sans doute d'un imaginaire ressentant le positionnement italien comme moins légitime. Par contre, l'énonciateur-traducteur français tend à mettre en valeur ses propres idées et à proposer ses solutions, sentant son positionnement comme légitime et autoritaire ${ }^{17}$. Cela lui permet de privilégier des verbes à visée incitative comme le verbe «demande», qui est le verbe le plus utilisé pour introduire les dispositions des rapports analysés (tableau 6) :

\begin{tabular}{ll}
\hline Fr. & It. \\
\hline Demande (206) & Invita (135) \\
\hline Souligne (147) & Chiede (131) \\
\hline Invite (128) & Sottolinea (110) \\
\hline Estime (41) & Ritiene (44) \\
\hline
\end{tabular}

Tableau 6 : Les verbes les plus utilisés pour introduire les dispositions des rapports d'initiative

À la différence du français, l'italien privilégie l'invitation (invita) à l'engagement de la demande, cette dernière ne venant qu'à la deuxième place (chiede). Les autres équivalents verbaux (souligne/sottolinea; estime/ritiene) ne montrent pas de désalignements significatifs à des fins d'analyse contrastive.

\section{Modalités d'ancrage énonciatif}

Si les paragraphes de la proposition de résolution sont le lieu idéal pour observer la prescription et l'engagement, l'exposé des motifs (cf. supra, fig. 1) permet au rapporteur de développer et d'expliciter les raisons qui justifient le positionnement pris et qui motivent les sollicitations faites aux acteurs de manière à les poser comme résolutives par rapport au problème soulevé. En tant que

17. Au sujet des attitudes différentes des traducteurs français et italiens, cf. la notion d' "éthos de la langue» (Raus, 2007, p.321-328). Pour le discours polémique / autoritaire, voir Puccinelli Orlandi, 1984. 
texte « hybride» (Devriendt et Monte, 2015, p. 71), il fournit des explications et des informations à des fins argumentatives ${ }^{18}$, afin de valider les actions prescrites, sollicitées ou souhaitées dans les paragraphes de la proposition de résolution. C'est donc le lieu idéal pour observer les modalités d'ancrage énonciatif.

Remarquons pourtant que l'ancrage temporel est peu fréquent et inscrit le rapport dans un contexte générique : en français, actuellement est utilisé quinze fois et sert, entre autres, à introduire les chiffres en «argument d'autorité » (Bessis et Remillon, 2012, p. 85); de nos jours n'est utilisé que trois fois. Les équivalents italiens attualmente/oggi (giorno) sont utilisés de la même manière.

Au plan spatial, le seul déictique présent, ici, est un hapax et renvoie au rapport, ce qui est confirmé par la traduction italienne nella presente rassegna (dans cette liste, pour renvoyer à la liste présente dans le rapport).

Concernant l'énonciateur, le rapporteur peut inscrire sa présence dans l'exposé, mais il le fait normalement à la troisième personne. En français, rares sont en effet les cas où il utilise les pronoms et adjectifs de la personne : je n'apparaît que comme hapax (Svensson, 2011, p.13), et votre, qui normalement est l'équivalent de l'anglais your dans l'expression your rapporter, est présent trois fois (Carlshmare, 2005 ; De Keyser, $2006{ }^{19}$; Svensson, 2011). Remarquons que dans ces cas, le traducteur italien intervient pour normaliser le texte à la nonpersonne, en utilisant l'expression la relatrice (la rapporteure) ou l'équivalent métonymique la relazione (le rapport). Même ce rapport est traduit en italien de manière désancrée par la relazione (le rapport) et non par questa relazione/ la presente relazione (ce rapport). La tendance italienne est donc de réaliser le maximum de dépersonnification et de désancrage, ce qui contribue encore plus à «la neutralité du constat» (Guilbert 2013, p. 300).

Le seul ancrage qui est suffisamment fréquent en français se fait par nous/ notre pour traduire l'anglais us/our. Le nous de majesté est présent seulement une fois pour renvoyer à la rapporteure (Karamanou, 2004, p. 16); les autres types de nous renvoient soit aux parlementaires soit à une collectivité générique, comme nous constatons par le tableau 7 (c'est nous qui utilisons les italiques) 20 :

18. Signalons que les marques de la description sont normalement absentes: les verbes à l'imparfait sont rares et le présent (non descriptif) reste le temps le plus utilisé. Par contre, on peut parfois retrouver des structures narratives contribuant au récit historique (voir les exposés des motifs d'Eriksson, 2004 et De Keyser, 2006). D'ailleurs, le Guide pratique commun pour la rédaction des textes législatifs de l'UE (2014) donne la possibilité de rappeler le contexte historique de l'acte juridique.

19. Précisons que le projet de rapport de la Belge De Keyser pourrait avoir été rédigé en français.

20. Nous nous demandons si la forte représentation des rapporteures suédoises dans l'emploi du pronom nous est due au hasard. 


\begin{tabular}{|c|c|c|}
\hline Rapport & Énoncé & Type de nous \\
\hline $\begin{array}{l}\text { Eriksson, } \\
2004\end{array}$ & $\begin{array}{l}\text { Les «spam» faisant de la publicité et la pornographie nous } \\
\text { envahissent... }\end{array}$ & Collectif \\
\hline $\begin{array}{l}\text { Carlshamre, } \\
2005\end{array}$ & $\begin{array}{l}\text {...demeurent dans l'UE, comme nous pouvons le constater... } \\
\text { Il est bien connu que les enfants ne font pas ce que nous } \\
\text { disons mais ce que nous faisons }\end{array}$ & $\begin{array}{l}\text { Pluriel } \\
\text { Collectif }\end{array}$ \\
\hline $\begin{array}{l}\text { De Keyser, } \\
2006\end{array}$ & ...alors que l'histoire nous donne tort? & $\begin{array}{l}\text { Pluriel/ } \\
\text { Collectif }\end{array}$ \\
\hline $\begin{array}{l}\text { Svensson, } \\
2011\end{array}$ & $\begin{array}{l}\text { Il est déplorable de constater que nous ne sommes... nous } \\
\text { devons rompre le silence... Nous devons réclamer un large } \\
\text { éventail de mesures... }\end{array}$ & $\begin{array}{l}\text { Pluriel } \\
\text { Pluriel }\end{array}$ \\
\hline $\begin{array}{l}\text { Carlshmare, } \\
2008\end{array}$ & $\begin{array}{l}\text { À ceux qui veulent considérer la prostitution... nous deman- } \\
\text { dons... nous demandons... nous demandons : comment } \\
\text { allons-nous... mais surtout comment allons-nous...? }\end{array}$ & $\begin{array}{l}\text { Majesté (?)/ } \\
\text { Pluriel }\end{array}$ \\
\hline $\begin{array}{l}\text { Honeyball, } \\
2014\end{array}$ & $\begin{array}{l}\text { Nous pouvons être certains d'une chose : la prostitution et } \\
\text { l'exploitation sexuelle sont incontestablement des questions } \\
\text { liées au genre }\end{array}$ & Pluriel \\
\hline
\end{tabular}

Tableau 7 : L'utilisation du nous pluriel et collectif dans l'exposé des motifs des rapports d'initiative français

Les équivalents italiens des énoncés du tableau 7 privilégient la nonpersonne, en optant pour l'indéfini Chi (Qui), l'impersonnel Si (On), les tournures passive ou impersonnelle ("quello che è certo », ce qui est certain). La pronominalisation dans le modal nous devons, qui n'apparaît que dans le rapport de la Suédoise Eva-Britt Svensson, est transformée en italien soit dans la tournure passive ("sono necessarie molte tipologie di misure ») soit dans la non-personne («la relatrice ritiene necessario rompere il silenzio»). Dans ce dernier cas, le nous pluriel français de l'énoncé «nous devons rompre le silence », qui est déjà dans la version anglaise (« we must break the silence»), est interprété en italien comme étant un nous de majesté, qui ne renvoie donc qu'à la rapporteure («la relatrice ritiene... »), en désengageant ainsi les autres députés.

En tant qu'ancrage à l'espace-temps actuel, l'expression notre/nos société-s (en anglais our society-ies) résume en gros l'utilisation de cet adjectif possessif dans le corpus français. Il s'agit du seul cas où l'ancrage de l'énonciateur est présent non seulement dans l'exposé des motifs mais aussi dans toutes les autres parties du rapport (considérants, paragraphes), comme on peut le voir à l'aide du tableau 8 : 


\begin{tabular}{ll}
\hline Rapport & Énoncé \\
\hline $\begin{array}{l}\text { Carlshamre, } \\
2005\end{array}$ & $\begin{array}{l}\text { [paragraphe } 1 \text { des dispositions]... la considérer [la violence domestique] } \\
\text { comme une violation des droits de l'homme qui reflète l'inégalité des rap- } \\
\text { ports de pouvoir dans notre société }\end{array}$ \\
\hline $\begin{array}{ll}\text { De Keyser, } \\
2006\end{array}$ & $\begin{array}{l}\text { [exposé des motifs] Les structures inégalitaires de nos sociétés confinent } \\
\text { les femmes dans des rôles traditionnels ou des postes subalternes }\end{array}$ \\
\hline $\begin{array}{l}\text { Svensson, } \\
2011\end{array}$ & $\begin{array}{l}\text { [considérants] considérant... que cette violence est liée à la répartition iné- } \\
\text { gale du pouvoir entre les femmes et les hommes au sein de notre société }\end{array}$ \\
\hline
\end{tabular}

Tableau 8 : Le paradigme de notre/nos société-s dans les rapports d'initiative français

Dans ces cas, nous constatons que la personne est aussi utilisée dans la traduction italienne (nostra/nostre società). Cela ne nous étonne pas vraiment, vu que l'ancrage reste générique, renvoyant à nos sociétés occidentales comme la rapporteure belge Véronique De Keyser le précise (2006, p.15), et destiné à réitérer le déjà-là naturalisé (Guilbert, 2013, p.11) pour lequel les inégalités («inégalité des rapports», "structures inégalitaires», "répartition inégale du pouvoir ») sont la cause des problèmes sociaux (violence à l'égard des femmes, rôles de genre).

Le tableau 8 permet également de remarquer la présence d'un véritable écho discursif qui caractérise le dit de Carlshamre (2005) et celui de Svensson (2011). Le lien entre la violence et les rapports inégaux de pouvoir dans notre société tend à créer un amalgame. Il arrive ainsi de trouver des segments textuels entiers qui sont repris par les rapporteurs, l'intertexte restant une caractéristique essentielle de ce type de documents (Raus, 2010, p.122). C'est justement cette notion qui va nous permettre de poser la question plus générale de la décontextualisation par rapport au contexte référentiel. La mise en discussion de l'amalgame femme-victime dans le rapport de l'Hongroise Zita Gurmai (tableau 9) en est un exemple révélateur.

Fr. (9) estime que les femmes dans les Balkans, qui ont été victimes de la guerre, ne devraient plus être considérées uniquement comme des victimes de guerre, mais plutôt comme des protagonistes dans la stabilisation et la résolution des conflits; (Exposé des motifs) Les femmes ne devraient plus être considérées uniquement comme des victimes de guerres et de conflits, mais elles peuvent et doivent être actrices de la paix et de la stabilisation. À cette fin elles doivent occuper des postes de responsabilité...

It. (9) ritiene che le donne nei Balcani, che hanno subito la guerra, non dovrebbero più essere considerate soltanto come vittime di guerra, quanto piuttosto come fautrici della stabilizzazione e risoluzione di conflitti; (Motivazione) Le donne non dovrebbero più essere viste soltanto come vittime di guerre e conflitti ma possono e devono avere il ruolo di fautrici di pace e stabilizzazione. Al fine di contribuire a questo processo devono rivestire posizioni decisionali...

Tableau 9: La contestation de l'amalgame femme-victime dans le rapport de Zita Gurmai (2008) 
Remarquons d'abord le fait que le positionnement affiché par le verbe d'opinion au paragraphe 9 servant à défendre la position claire du Parlement européen concernant le refus de l'amalgame femme-victime ne trouve pas de vraies justifications dans l'exposé des motifs, qui se limite à reprendre le dit. Le discours produit l' "effet d'évidence» (Guilbert, 2009) non seulement par la tautologie du propos mais aussi par la généralisation de la source d'énonciation : ce n'est pas la rapporteure qui «estime» ou qui défend son idée de manière explicite dans l'exposé, mais c'est le Parlement européen qui prend une position explicite une fois que le rapport sera approuvé.

Cette généralisation ne concerne pas que l'ancrage du dit, comme on peut le voir par la reprise du texte de Gurmai dans le rapport de la Roumaine Norica Nicolai (tableau 10) :

Fr. (28) estime que les femmes qui ont été victimes de la guerre ne devraient plus être considérées uniquement comme des victimes de guerre, mais plutôt comme des protagonistes dans la stabilisation et le règlement des conflits

It. (28) è del parere che le donne vittime della guerra non dovrebbero più essere considerate soltanto come vittime di guerra, quanto piuttosto come fautrici della stabilizzazione e risoluzione di conflitti

Tableau 10 : La reprise du rapport Gurmai (2008) dans le rapport Nicolai (2011)

En effet, dans la reformulation de 2011, l'opinion présentée ne concerne pas que les Balkans mais acquiert une portée générale. À la différence du désancrage énonciatif, qui implique la seule absence de déictiques, la décontextualisation gomme tout point de repère référentiel, ce qui produit une généralisation maximale du dit en français comme en italien.

Remarquons, pour finir, que la reprise discursive du segment textuel de Gurmai (paragraphe 9) dans Nicolai (paragraphe 28) présente quelque écart dans les versions françaises («règlement/résolution») et italiennes («che hanno subito la guerra/vittime della guerra»), dû au fait que les traducteurs du rapport Nicolai retraduisent de l'original sans tenir compte de la traduction déjà existante du segment en question. Dans les rapports anglais, par contre, qui sont sans doute les originaux ${ }^{21}$, les paragraphes 9 et 28 sont identiques («women [in the Balkans], who have been victims of war, should no longer be seen only as war victims but rather as actors of stabilisation and conflict resolution »), à l'exception du verbe introducteur (considers devient takes the view that dans la reprise) et les désalignements caractérisent plutôt l'exposé des motifs («Women should no longer only be seen as victims of wars and conflicts

21. Gurmai et Nicolai utilisent l'anglais lorsqu'elles présentent des amendements aux rapports parlementaires (voir, entre autres, le rapport Eriksson 2011). Rappelons que les amendements sont des documents privilégiés pour retrouver les langues de travail des députés, puisqu’ils explicitent la langue dans laquelle chaque amendement est proposé. 
but they can and should be actors of peace and stabilisation »), ce qui confirme que normalement l'exposé est la partie du texte la plus libre des rapports parlementaires du point de vue de la rédaction et de la traduction.

\section{En guise de conclusion}

Bien que la prostitution soit un sujet polémique, la validation de la prescription et en général de tout positionnement passe par la propension au désancrage énonciatif, plus évident en italien, et à la décontextualisation lors de la reprise des segments intertextuels, ce qui contribue à l'effet d'évidence dans les rapports en français comme en italien. Cette tendance à la neutralisation est donc une caractéristique commune de ce genre discursif indépendamment de la langue.

Ce qui varie dans les deux langues est plutôt la présence d'effets performatifs décalés. En ce sens, la version italienne reste plus euphémique que la version française, atténuant les visées prescriptive et incitative, comme permet de le constater la tendance du traducteur italien à :

- privilégier le verbe modal dovere (devoir) au conditionnel;

- utiliser moins fréquemment certains verbes à visée incitative, par exemple chiede (demande);

- marquer le dit par des stratégies polémiques d'ouverture aux autres acteurs, ce qui dénote un sentiment d'illégitimation ;

- généraliser davantage par un désancrage énonciatif total, le seul ancrage restant le nous à valeur fortement générique de l'expression nostra/e società (notre/nos société-s).

Les deux versions linguistiques finissent donc par réaliser différemment la dynamique discursive de la prescription/incitation et du lissage énonciatif, en engageant différemment les acteurs concernés. La version italienne reste plus faible par rapport à l'engagement demandé et tend à la dépersonnification maximale du dit; la version française marque davantage la prescription et l'incitation et finit par restituer un dit où l'on arrive parfois à distinguer la présence de voix précises.

Ces différences pourraient trouver une explication partielle dans le fait que, comme pour n'importe quel autre genre discursif qui est forcément lié au contexte de production, le rapport d'initiative implique l'adaptation aux styles et aux normes de rédaction nationaux. En ce sens, les tendances retracées pourraient être l'indice des pratiques d'adaptation normalement utilisées pour ce type de documents dans les langues nationales.

Cependant, le positionnement ressenti comme moins légitime du traducteur italien, qui a des répercussions sur l'efficacité pragmatique de son dit, le porte à faire des choix précis, comme par exemple, dans le cas de l'énoncé peu 
lisible parce que calqué sur l'anglais ou lors de l'omission de certains acteurs et de la reformulation d'autres. Inversement, le traducteur français n'a pas peur de ne pas neutraliser la connotation ou de rendre son texte lisible en affichant clairement un positionnement précis.

En ce sens, l'attitude des traducteurs semble fort proche de celle des députés qui sont leurs homologues au Parlement européen. Dans les explications de votes aux rapports concernés, les députés italiens se montrent d'ordinaire favorables aux documents et soulignent leur alignement sur les propositions approuvées, tandis que les députés français insistent sur l'apport de leur propre groupe politique et de la France à la «production de l'Europe» (Trimithiotis, 2013, p. 280). À ce propos, les commentaires de vote au rapport Svensson signés par Karima Delli et par Sylvie Guillaume sont exemplaires, le premier soulignant l'apport d'un amendement approuvé qui a été proposé par le groupe politique de Delli, et le deuxième engageant fortement le Conseil et la Commission «qui ne peuvent ne pas manquer cette étape indispensable » pour aider les femmes victimes de violence.

Il nous semble alors possible d'affirmer que la traduction et, par-là, la présence d'une double énonciation, sont des éléments qui contribuent à l'ouverture colingue du discours parlementaire (Raus, 2015, p.130) et qui permettent au dit du Parlement européen de ne pas se figer dans un discours « instituant» (Oger et Ollivier-Yaniv, 2003b, p. 2) et de rester constitutivement polyphonique, fruit «d'un processus de production collective» (Ibid., p.10).

\section{Références}

Anthony Laurence, 2014, AntPConc (Version 1.1.0) [Computer Software], Tokyo, Waseda University, 〈http://www.laurenceanthony.net / (consulté le 24 mars 2017).

Bessis Franck, Remillon Delphine, 2012, "Déchiffrer l'économie au Parlement. La place des arguments chiffrés dans les débats sur les semaines de 40 et 35 heures ", Mots. Les langages du politique, $\mathrm{n}^{\circ} 100, \mathrm{p} .75-89$.

CABAsino Francesca, 2001, Formes et enjeux du débat public. Discours parlementaire et immigration, Rome, Bulzoni.

CALIENDo Giuditta, 2004, «nEUlogisms : produttività lessicale del linguaggio comunitario»,Inter@lia, n³0, p.8-10.

Cussò Roser, Gobın Corinne, 2008, «Du discours politique au discours expert : le changement politique mis hors débats? », Mots. Les langages du politique, $\mathrm{n}^{\circ}$ 88, p. 5-11.

DeVRIEnd Emilie, MonTE Michèle, 2015, «L'exposé des motifs : un discours d'autorité. Le cas des lois françaises de 2003, 2010 et 2014 Sur les retraites», Mots. Les langages du politique, $\mathrm{n}^{\circ} 107$, p. 67-84.

Europe : Parlement européen, 2004, Règlement du Parlement européen (16 é éditionjuillet2004), 〈http://www.cvce.eu/content/publication/2005/6/3/0535a8383799-44ad-a6cb-aa6dfo139ob7/publishable_fr.pdf> (consulté le 24 mars 2017). 
Europe : PARlement européen, 2009, Règlement du Parlement européen (7e législaturejuillet 2013), <http://www.cvce.eu/content/publication/2014/2/12/91a79c75-beae4b82-aac2-bo86de3cf8f5/publishable_fr.pdf> (consulté le 24 mars 2017).

GUILBERT Thierry, 2009, "La "réalité" de la presse écrite et l'éthique de l'analyse du discours », Le rapport éthique au discours, C. Guérin, G. Siouffi, S. Sorlin éd., Berne, Peter Lang, p. 289-302.

GUILBERT Thierry, 2013, "Discours d'évidence. Construction discursive des normes et des connaissances", Normativités du sens commun, C. Gautier et S. Laugier éd., Paris, PUF, p. 275-300; disponible sur Internet <https://halshs.archives-ouvertes. $\mathrm{fr} /$ halshs-00813896> (consulté le 24 mars 2017).

Hermand Marie-Hélène, 2014, «Le discours eurorégional. Indices convergents de légitimation d'un espace institutionnel », Mots. Les langages du politique, $\mathrm{n}^{\circ} 106, \mathrm{p} .71-86$.

Ho-Dinh Océane, ValetTe Mathieu, 2014, «Analyse différentielle des discours de prévention du VIH : textes institutionnels et textes informels en français et en vietna-

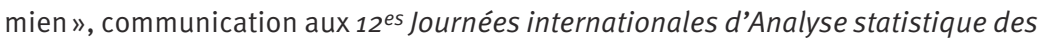
données textuelles (JADT) 〈http://lexicometrica.univ-paris3.fr/jadt/jadt2014/01ACTES/23-JADT2014.pdf> (consulté le 24 mars 2017).

KOREN Roselyne, 2008, «Pour une éthique du discours : prise de position et rationalité axiologique », Argumentation et analyse du discours, n² 1, 〈http://aad.revues. org/263> (consulté le 24 mars 2017).

KRIEg-PlAnqUe Alice, 2012, Analyser les discours institutionnels, Paris, A. Colin.

Krieg-Planque Alice, Oger Claire, 2010, «Discours institutionnels. Perspectives pour les sciences de la communication », Mots. Les langages du politique, $n^{\circ}$ 94, p. 91-96.

LEONCINI BARTOLI Antonella, 2011, « Les normes de traduction au sein de l'Union européenne : "unité dans la diversité" ou "diversité dans l'unité"?", SCOLIA, n² 25 , p. $225-238$.

Maingueneau Dominique, 1991, L'analyse du discours, Paris, Hachette.

MAINGUENEAU Dominique, 2002, "Les rapports des organisations internationales: un discours constituant? ", Les mots du pouvoir. Sens et non-sens de la rhétorique internationale, G. Rist éd., Paris, PUF; Genève, Institut universitaire d'études du développement, p.119-132; disponible sur Internet <http://books.openedition. org/iheid/2443> (consulté le 24 mars 2017).

MaIngueneau Dominique, 2007, "Genres de discours et modes de généricité », Le français d'aujourd'hui, $\mathrm{n}^{\circ} 159$, p. 29-35.

MaINGUENEAU Dominique, [2015], «Typologie des genres de discours», disponible sur Internet : 〈http://dominique.maingueneau.pagesperso-orange.fr/pdf/Typologiedes-genres-de-discours.pdf> (consulté le 24 mars 2017).

Maingueneau Dominique, Charaudeau Patrick, 2002, Dictionnaire d'analyse du discours, Paris, Éd. du Seuil.

MONTE Michèle, OGER Claire éd., 2015, "Discours d'autorité : des discours sans éclat(s) ?», Mots. Les langages du politique, $\mathrm{n}^{\circ} 107$.

Oger Claire, Ollivier-Yaniv Caroline, 2003a, "Analyse du discours institutionnel et sociologie compréhensive : vers une anthropologie des discours institutionnels», Mots. Les langages du politique, $\mathrm{n}^{\circ} 71$, p. 125-144. 
Oger Claire, Ollivier-Yaniv Caroline, 2003b, «Du discours de l'institution aux discours institutionnels : vers la constitution de corpus hétérogènes», communication au Xe Colloque bilatéral franco-roumain, CIFSIC Université de Bucarest, 28 juin-3 juillet 2003, disponible sur Internet : 〈http://archivesic.ccsd.cnrs.fr/sic_00000717〉 (consulté le 24 mars 2017).

Oger Claire, Ollivier-Yaniv Caroline, 2006, «Conjurer le désordre discursif. Les procédés de "lissage" dans la fabrication du discours institutionnel», Mots. Les langages du politique, $\mathrm{n}^{\circ} 81$, p. 63-77.

Paveau Marie-Anne, 2010, "Interdiscours et intertexte. Généalogie scientifique d’un pair de faux jumeaux», Linguistique et littérature : Cluny, 40 ans après [ouvrage collectif issu du colloque international tenu du 29 au 31 octobre 2007 à Besançon], D. Ablali et M. Kastberg-Sjöblom éd., Besançon, PUFC, p. 93-105.

PuCCINELLI OrLANDI Eni, 1984, «Typologie du discours et règles de la conversation», Langage et société, vol. 29, $\mathrm{n}^{\circ}$ 1, p. 3-25; disponible sur Internet : 〈http://www.persee.fr/doc/lsoc_0181-4095_1984_num_29_1_1998> (consulté le 24 mars 2017).

RAus Rachele, 2007, «La terminologie des Bourses italienne et française: quelle relation à la langue anglaise », Langue, économie, entreprise. Le travail des mots [Textes issus d'un colloque organisé à l'Université Paris 3, du 13 au 15 novembre 2003], I. Behr, D. Hentschel, M. Kauffmann et A. Kern éd., Paris, Presses de la Sorbonne nouvelle, 2007, p. 311-325.

RAus Rachele, 2010, «Terminologia comunitaria e di settore nelle relazioni parlamentari », Multilinguismo e terminologia nell'Unione europea, R. Raus éd., Milan, Hoepli, p. 115-156.

Raus Rachele, 2013, La terminologie multilingue. La traduction des termes de l'égalité H/F dans le discours international, Bruxelles, De Boeck.

Raus Rachele, 2015, "Types de contre-discours et remaniements "codiscursifs" : l'inscription du dit d'ATTAC et du LEF dans les rapports du Parlement européen sur les femmes (2004-2012)», Semen, n³ 39, p. 115-134.

SAURUgger Sabine, 2003, "Les groupes d'intérêts entre démocratie associative et mécanismes de contrôle», Raisons politiques, n² 2, p. 151-169.

SCHulze Erika et alii, 2014, L'exploitation sexuelle et la prostitution et leurs conséquences sur l'égalité entre les femmes et les hommes, Bruxelles, Office des publications du Parlement européen, disponible sur Internet : 〈http://www.europarl.europa.eu/ RegData/etudes/etudes/join/2014/493040/IPOL-FEMM_ET(2014)493040_FR.pdf> (consulté le 24 mars 2017).

Steinberger Ralf et alii, 2014, An Overview of the European Union's Highly Multilingual Parallel Corpora, disponible sur Internet : 〈https://ec.europa.eu/jrc/sites/jrcsh/ files/2014_08_LRE-Journal_JRC-Linguistic-Resources_Manuscript.pdf $>$ (consulté le 24 mars 2017).

ToUTAIN Ghislaine, 2013, Pour un parlement européen paritaire en 2014. Dix actions à conduire par les partis progressistes européens poury parvenir, Jean Jaurès Fondation. URL : 〈http://www.feps-europe.eu/en/publications/details/36〉 (consulté le 24 mars 2017). 
TRIMITHIOTIS Dimitris, 2013, La production des discours électoraux comme configuration des mythes politiques européens : une analyse comparative des programmes électoraux européens, français et chypriotes pour les élections du Parlement européen de 2009, Thèse de doctorat : Sociologie, Université d’Aix-Marseille «http:// www.theses.fr/2013AIXM3052> (consulté le 24 mars 2017).

TRON Giorgio, 2010, «Alcune postille sulla traduzione del modale should nei "considerando" degli atti normativi », Inter@lia, n46, p.18-22.

VETTERS Carl, 2004, "Les verbes modaux pouvoir et devoir en français ", Revue belge de philologie et d'histoire, vol.22, $\mathrm{n}^{\circ} 3$, p. 657-671.

\section{Sources}

Nous avons transcrit les seuls titres des versions françaises des rapports analysés. Notre corpus inclut également les versions italiennes et anglaises de ces documents.

CARLSHAMRE Maria, 2005, Rapport sur la situation actuelle de la lutte contre la violence à l'égard des femmes et toute action future (2004/2220(INI)).

CARLSHAMre Maria, 2008, Projet de rapport sur la prostitution et ses conséquences sur la santé des femmes dans les États membres (2007/2263(INI)).

DE KEYSER Véronique, 2006, Rapport sur la situation des femmes dans les conflits armés et leur rôle dans la reconstruction et le processus démocratique dans les pays en situation post-conflit (2005/2215(INI)).

ERIKSSON Marianne, 2004, Rapport sur les conséquences de l'industrie du sexe dans l'Union européenne (2003/2107(INI)).

Gurmal Zita, 2008, Rapport sur la situation des femmes dans les Balkans (2008/2119 $(\mathrm{INI}))$.

Honeyball Mary, 2014, Rapport sur l'exploitation sexuelle et la prostitution et leurs conséquences sur l'égalité entre les hommes et les femmes (2013/2103(INI)).

KARAMANou Anna, 2004, Rapport sur les femmes dans l'Europe du Sud-Est $(2003 / 2128(I N I))$.

MARtínez-Orozco Elena Valenciano, 2004, Rapport sur la situation des femmes issues de groupes minoritaires dans l'Union européenne (2003/2109(INI)).

Nicolal Norica, 2011, Rapport sur la situation des femmes en période de guerre (2011/2198(INI)).

PRETS Christa, 2005, Rapport sur des stratégies de prévention de la traite des femmes et des enfants vulnérables à l'exploitation sexuelle (2004/2216(INI)).

SVENSSON Eva-Britt, 2011, Rapport sur les priorités et la définition d'un nouveau cadre politique de l'Union en matière de lutte contre la violence à l'encontre des femmes (2010/2209(INI)). 